\title{
Apuntes para el repensamiento de la enseñanza de la Arquitectura. La cuestión epistemológica y la necesidad de una razón ampliada
}

\author{
Notes for rethinking the teaching of Architecture. The epistemological question and the need for an \\ expanded reason \\ Anotações para repensar o ensino da Arquitetura. A questão epistemológica e a necessidade de uma \\ razão ampliada
}

\author{
Juan J. Álvarez-Álvarez \\ Universidad Francisco de Vitoria. Madrid (España) \\ Escuela de Arquitectura
}

Álvarez-Álvarez, J. (2019). Apuntes para el repensamiento de la enseñanza de la Arquitectura. La cuestión epistemológica y la necesidad de una razón ampliada. Revista de Arquitectura (Bogotá), 21(2), 57-67. doi: http://dx.doi.org//0.14718/RevArq.2019.21.2.1917

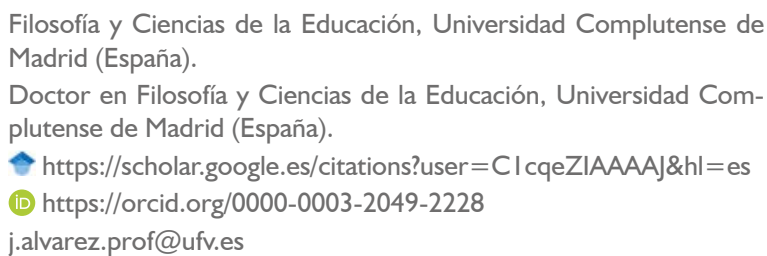

\section{Resumen}

Es necesario repensar la enseñanza de la Arquitectura de modo que pueda integrar armónicamente las tres dimensiones que siempre la han conformado (artística, técnica y científica) y evitar la presentación fragmentada de los saberes. A partir de indagaciones pedagógicas y mediante la sistematización de experiencias docentes de intercambio, se construye un sustento teórico que se soporta desde la filosofía de la ciencia, la tecnología y la antropología. Por tanto, se ocupa de la cuestión epistemológica y de la necesidad de abordar el repensamiento desde una razón ampliada, abierta a la pluralidad y riqueza de la realidad. Lo que se interpreta es que, aunque no todas las asignaturas permiten igual profundidad en el repensamiento, siempre es posible reconocer las posibilidades que las distintas disciplinas ofrecen, así como los límites que les impiden contribuir a que el ser humano avance en la búsqueda de verdades cada vez más profundas y trascendentes. De ese repensamiento se seguirán cambios en la labor docente, vista desde la enseñanza integrada y con sentido, y lejos de la adición de materias humanísticas como complemento a la formación del estudiante.

Palabras clave: ciencia y tecnología; educación superior; pedagogía y didáctica de la arquitectura; integración curricular; investigación proyectual.

\begin{abstract}
It is necessary to rethink the teaching of architecture such that it can harmoniously integrate the three dimensions that have always comprised it (art, technique, and science) and avoid the fragmented presentation of knowledge. Based on pedagogical research and the systematization of experiences of teaching exchanges, this article develops theoretical support from philosophy of science, technology, and anthropology. It addresses the epistemological question and the need to address rethinking from expanded reason, open to the plurality and richness of reality. The article considers although not all subject areas allow for an equally deep rethinking, it is always possible to recognize the possibilities different disciplines offer, as well as the limits that prevent them from contributing to mankind's' progress in the search for increasingly profound and transcendental truths. Changes in teaching follow from this rethinking, including integrated and meaningful teaching, and far from the addition of humanistic topics as a complement to students' training.
\end{abstract}

Keywords: Science and technology, higher education, pedagogy and teaching approach in architecture, curricular integration, project research.

\section{Resumo}

É necessário repensar o ensino da Arquitetura a fim de integrar, de forma harmônica, as três dimensões que a conformam (artística, técnica e científica) e evitar a apresentação fragmentada dos saberes. A partir de questionamentos pedagógicos e da sistematização de experiências docentes de intercâmbio, é construído o referencial teórico apoiado na filosofia da ciência, na tecnologia e na antropologia. Trata-se da questão epistemológica e da necessidade de abordar o repensamento a partir de uma razão ampliada, aberta à pluralidade e à riqueza da realidade. Embora nem todas as disciplinas permitam a mesma profundidade no repensamento, sempre é possível reconhecer as possibilidades que as diferentes disciplinas oferecem bem como os limites que lhes impedem contribuir para que o ser humano avance na busca de verdades cada vez mais profundas e transcendentes. Desse repensamento, virão mudanças no fazer docente, visto a partir do ensino integrado e com sentido, e longe da adição de matérias de humanas como complemento para formar o estudante.

Palavras-chave: ciência e tecnologia; educação superior; pedagogia e didática da arquitetura; integração curricular; pesquisa projetual.

\section{Introducción}

Este artículo se enmarca en los cursos de Introducción a las Humanidades dirigidos a los profesores de la Escuela Politécnica Superior de la Universidad Francisco de Vitoria (UFV), en la que el autor ha enseñado durante los últimos años diversas materias filosófico-teológicas, y ante la necesidad de aterrizar ciertas cuestiones relativas a la forma en la que podrían repensar sus disciplinas y el modo en el que se impartían, cuando comenzó a esbozarse este trabajo.

La propuesta que se plantea tiene que ver con la necesidad de un repensamiento de la educación universitaria, especialmente en las áreas científico-técnicas, para dotarla del carácter humanista y transdisciplinar que a menudo se echa de menos hoy; un enfoque que atenúe la propensión de nuestro tiempo a una especialización salvaje y al imperio absoluto de la razón instrumental, y que recupere, actualizándolos, los valores que siempre habían distinguido a la Universidad desde sus orígenes. Dicha propuesta se enmarca en la preparación y el desarrollo de una experiencia que, tras años de reflexión y pruebas, está poniéndose en práctica en la UFV de Madrid.

No se trata propiamente de un proyecto de investigación al uso, sino más bien de un programa teórico-práctico que atañe a la misión y la visión de dicha universidad, y a la forma en la que quiere encarar los nuevos retos que la razón y la fe han de afrontar en nuestros días, particularmente en el entorno de la enseñanza universitaria. 


\section{Metodología}

El autor del presente texto fue enviado como visiting research y como fellow respectivamente, durante un año entero a dos universidades estadounidenses: Assumption College, en Worcester (Massachusetts) y el Center of Catholic Studies de la University of Saint Thomas, en Saint Paul (Minnesota). En su ánimo estaba hacer contacto con algunos proyectos más o menos similares que dichas universidades pretendían implementar. Sobre todo, en relación con el segundo de los centros mencionados, la colaboración entre ambas entidades ha deparado ya algunos frutos, aunque el contexto y los intereses propios de las instituciones no coincidan plenamente.

Siendo este el marco de referencia del presente estudio, el enfoque desde el que aquí se quiere iluminar concretamente la enseñanza de la Arquitectura, más allá de algunas citas que intentan introducir el tema, no tiene tanto que ver, sin embargo, con dicho proyecto, cuanto con los cimientos especulativos sobre los que sostiene, fundamentos que parecen adecuados también para otras universidades politécnicas y, de forma particular, para otras escuelas de Arquitectura.

En su origen, por tanto, venía estimulado más por motivos de índole pedagógica que especulativa. No obstante, inmediatamente, emergió la conveniencia de dotar de un sustento teórico dichos cursos y, de modo consiguiente, la exigencia de una reflexión ad hoc, alimentada de la propia experiencia investigadora ${ }^{1}$ y docente, así como de múltiples lecturas que abarcaban campos en principio tan aparentemente distantes como la epistemología, la filosofía de la ciencia y de la tecnología, la antropología y, obviamente, la propia arquitectura.

\section{Resultados}

A la hora de empezar a plasmar por escrito el resultado de dicha reflexión, conviene, sin embargo y antes que cualquier otra cosa, advertir acerca de un error que haría fracasar esta tarea desde su inicio: interpretar que el repensamiento de la enseñanza de la Arquitectura $y$, por ende, la renovación interna de las disciplinas que la conforman en el ámbito de la docencia universitaria consistiera en el añadido de un mayor número de créditos u horas lectivas pertenecientes a materias humanísticas de literatura, historia, arte, filosofía o incluso teología, materias que vendrían a yuxtaponerse con las asignaturas propias del grado o carrera con el objetivo de complementar la formación del estudiante. Dicho enfoque, lejos de contribuir a una enseñanza integrada y con sentido, contribuiría a una presentación aún más fragmentada de los

1 Para un análisis de cómo enfocar hoy la investigación humanística en una universidad católica, véase Álvarez-Álvarez (2011). saberes y acrecentaría su dispersión en la mente del alumno. Por tanto: "El repensamiento de las asignaturas requiere una mirada distinta que brota de lo que se ha llamado 'razón ampliada', busca la unidad del saber, tiene su base en las preguntas fundamentales (antropológica, ética, epistemológica y de sentido), y, por supuesto, pone en juego tanto al profesor como al alumno" (Lacalle, 2014, p. 25).

Para encaminar la tarea, lo que se hace es partir de unos presupuestos que parecen fácilmente accesibles a cualquiera:

Por una parte, la caracterización del ser humano (y, por ende, del universitario) como un buscador de la verdad en todos los planos y niveles de su vida (desde los que tienen que ver con las dimensiones más utilitarias o pragmáticas hasta los referidos a cuestiones últimas relacionadas con la pregunta por el sentido de la existencia). Por otra, la concepción de que la verdad que el hombre busca tiene un carácter multidimensional o, por así decir, es semejante a una sinfonía compuesta de notas y acordes distintos que se armonizan para la composición de una obra final unitaria y consistente, que transciende la fragmentariedad propia de las diversas disciplinas consideradas en forma multi o pluri-disciplinar. (Álvarez-Álvarez, 2018, p. 101)

Teniendo en cuenta estos presupuestos, no puede resultar extraño que la UFV se plantee, en los documentos que plasman el espíritu de su idea de universidad, la necesidad de no conformarse con la capacitación profesional y técnica de los estudiantes, sino atender de forma integral a su búsqueda y a las múltiples preguntas que les impulsan, y que también inquietan y motivan a los docentes, los investigadores, el personal de administración y servicios, e incluso a los padres de esos mismos estudiantes.

En concreto, respecto de las enseñanzas técnicas el documento-misión de la UFV dice lo siguiente:

Porque es más que una escuela profesional [...] la Universidad no debe autolimitarse al descubrimiento de conocimientos y técnicas útiles, ni tampoco puede olvidar que la aplicación de los conocimientos no es neutral con respecto al hombre, ni los medios para obtenerlos son indiferentes. Más bien debe tener siempre presente que el cometido de la técnica es hacer posible que se realice el proyecto en que consiste la vida humana y que la profesión o tarea a realizar son simplemente el marco de posibilidades para realizar una auténtica obra personal. (Universidad Francisco de Vitoria, s. f., p. 3)

Y, más adelante, se añade:

La especialización es una exigencia del método científico [...]. Empero, si la Universidad no es más que un océano de saberes fragmentados será difícil que mantenga un rumbo [...]. Hace falta una síntesis que integre el fragmento en el todo. Hace falto 'algo más' que el conocimiento puramente útil o simplemente interesante. (Universidad Francisco de Vitoria, s. f., p. 8) 
Por su parte, el ideario de la Escuela de Arquitectura especifica que

\begin{abstract}
... a la hora de enfrentarse a un proyecto arquitectónico, un programa urbanístico o el diseño de una estructura, esto es, cuando el arquitecto afronta la tarea de aportar soluciones habitables adecuadas a la persona y su entorno, ha de considerar todas aquellas realidades que de una forma directa e indirecta están afectadas por su obra. Un arquitecto con una mirada acertada descubre ámbitos que, partiendo del mismo campo de la Arquitectura, lo trascienden. Si su trabajo pretende responder a las necesidades del hombre, considerando las circunstancias particulares de cada persona, el arquitecto entonces debe tener en cuenta cuestiones antropológicas, estéticas, sociales, culturales, económicas, políticas, demográficas, geográficas... que no son, ni pueden ser aleatorias. (Samarán y Parada, 2013, p. 15)
\end{abstract}

Cada una de las cuestiones que aquí se mencionan merecería por sí sola una reflexión, pues influyen o interaccionan con el proceso de enseñanza y aprendizaje de la profesión en el que el estudiante de Arquitectura se halla inmerso durante su periodo de formación. Este trabajo, por mor de la brevedad, se ocupará únicamente de una de ellas, a la que pocas veces se hace referencia: la cuestión epistemológica (Figuras 1 y 2) y a la necesidad de abordar el repensamiento desde una razón ampliada, abierta a la pluralidad y riqueza de la realidad. En ello, precisamente, puede cifrarse su empeño y su conclusión fundamental ${ }^{2}$.

\section{¿Qué es la arquitectura?}

Se han intentado muchas definiciones de lo que pueda ser la arquitectura, pero puesto que lo que aquí se pretende no tiene que ver, al menos directamente, con esta cuestión, bastará con decir que, desde la opinión personal, la arquitectura es, a la vez, arte, técnica y ciencia. ¿Qué se quiere significar con ello?

Por arte se puede entender, en general, el conjunto de reglas que se exigen para hacer bien una cosa. Visto así, viene a ser sinónimo de destreza o habilidad. Ahora bien, si se habla de "bellas artes", se han de añadir a este "buen hacer" los elementos propios de la creatividad humana y de la búsqueda de la belleza.

La técnica puede caracterizarse como el conjunto de procedimientos, instrumentos y recursos de los que se sirve una ciencia o un arte en sus posibles aplicaciones prácticas. De ahí que, a diferencia de la ciencia, la técnica no aspira a desvelar o explicar el fondo de las cosas sino a proporcionar los medios más eficaces para actuar sobre ellas obteniendo así un cierto beneficio o utilidad.

2 Puede encontrarse una propuesta paralela del mismo autor para las Escuelas Superiores de Ingeniería Informática, en ese caso en lo tocante a la cuestión antropológica. (Álvarez-Álvarez, 2018)
La ciencia, por su parte, implica un cierto saber, un conocimiento sistemático, riguroso y bien fundado de las causas o los principios que explican o dan razón de un objeto determinado, que previamente se ha seleccionado para su análisis. Así concebida, puede enfocarse desde dos perspectivas: objetiva y subjetiva. Según aquella, un saber científico se constituye como un conjunto de verdades relativas a su objeto de estudio y lógicamente relacionadas entre sí de manera que conformen un sistema coherente y consistente; considerada desde un punto de vista subjetivo, en cambio, se referiría al conocimiento cierto (seguro) de las cosas, por sus causas o por sus leyes. Aunque, de acuerdo con su dimensión sapiencial, la investigación científica básica tiene valor por sí misma en la medida en que responde al natural deseo humano de saber. La ciencia tiene igualmente un valor añadido fundamental, aplicado desde el momento en que proporciona sus conocimientos a la técnica para los propósitos prácticos que esta fije. Ambas, ciencia y tecnología, deben estar al servicio del hombre.

Hechas estas sencillas consideraciones preliminares, ha de decirse que la Arquitectura es -simultáneamente- arte (una de las bellas artes), técnica y ciencia. (v) Figura 1. Ciudad rehabitada. Actividad dentro de la Semana de la Arquitectura

Fuente: Universidad Francisco de Vitoria, 2017.

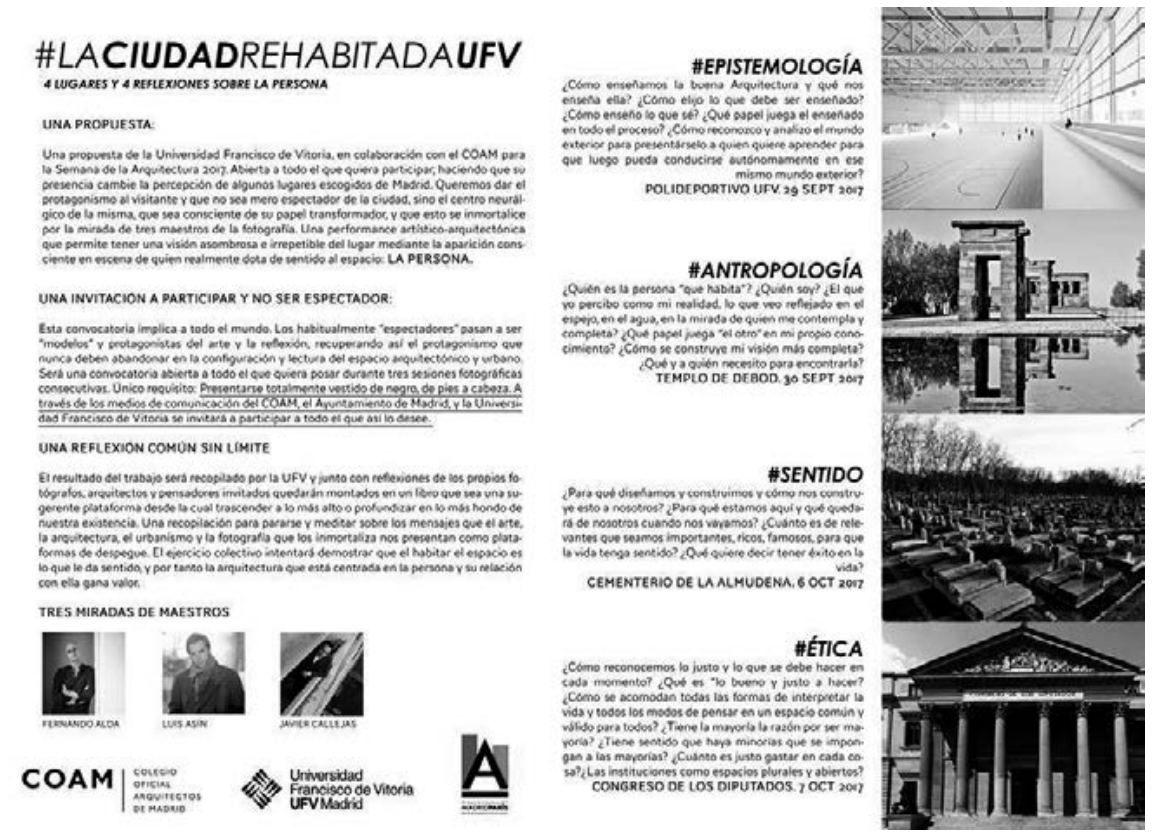

(7) Figura 2. Ciudad rehabitada Epistemología. Actividad dentro de la Semana de la Arquitectura Fuente: Universidad Francisco de Vitoria, 2017

\#EPISTEMOLOGÍA

¿Cómo enseñamos la buena Arquitectura y qué nos enseña ella? ¿Cómo elijo lo que debe ser enseñado? ¿Cómo enseño lo que sé? ¿Qué papel juega el enseñado en todo el proceso? ¿Cómo reconozco y analizo el mundo exterior para presentárselo a quien quiere aprender para que luego pueda conducirse autónomamente en ese que luego pueda conducirse autonomamente en ese POLIDEPORTIVO UFV. 29 SEPT 2017

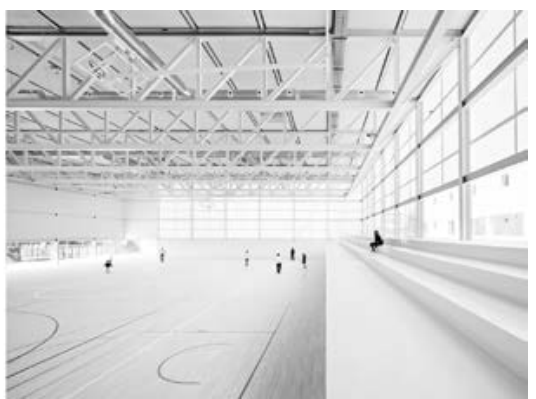


Es un "bello arte", en cuanto el arquitecto no puede dejar de lado la cuestión de la belleza en su quehacer cotidiano; amén de otras dimensiones, su trabajo tiene sin duda una dimensión estética. Por qué haya de ser así necesariamente se encuentra implícito en el hecho de que los espacios generados por el arquitecto tienen la finalidad de servir de hábitat al ser humano, considerado este en la totalidad de sus dimensiones, necesidades y aspiraciones. Solo el hombre, propiamente, habita un espacio (no se dice de las cosas o del resto de los seres vivos que sean habitantes de un lugar), y lo hace con todo su ser y rodeado de aquellos otros seres (vivos o inertes) que para él significan algo importante en el quehacer (profesional, lúdico, social y personal) que constituye su vida. Entre estos fines humanos a los que el arquitecto busca responder están, por supuesto, los que tienen que ver con su supervivencia en condiciones dignas, pero no podemos olvidar que esta dignidad no se reduce ni mucho menos al mero sobrevivir, ni siquiera a la sobrevivencia con altos niveles de calidad de vida, sino que incluye un deseo de verdad, de bien y justicia, de libertad y creatividad, en fin, de belleza.

En segundo lugar, es la arquitectura igualmente técnica pues para el arquitecto el conocimiento de su oficio y de las leyes que lo regulan - con el fin de poder ofrecer en todo momento las soluciones más adecuadas, viables, seguras y eficientes a los problemas que sus clientes le planteen- resulta de todo punto esencial. En la definición de arquitectura que se ha propuesto, este elemento estaría incluido en las acciones expresadas por los verbos proyectar, ordenar, planificar, construir, reformar o rehabilitar.

Y por último, la arquitectura es también ciencia, ciencia que versa sobre el espacio real y sobre lo que en él se puede generar para el fin buscado. Y lo es, especialmente, en el sentido de que su ejercicio precisa de un saber interdisciplinar y cada día más especializado. Es este último aspecto, el científico, el que convierte a la arquitectura en un saber susceptible de cátedra universitaria, pues si bien la parte técnica se puede y debe entrenar -hoy en día, además, con multitud de herramientas virtuales y simulaciones que acercan gráficamente la práctica y los materiales que necesita a sus consecuencias y resultados reales-, hay en cambio cuestiones más profundas, un modo de razonar y preguntar, y un método implícito en el hacer del arquitecto que no surge de, pero sí se nutre de la investigación continuada acerca de las causas de esta o aquella distribución del espacio, de la edificabilidad, de la resistencia de tal o cual material o de la planificación más oportuna para una urbe.

\section{Grandeza y límite de la arquitectura}

Cada uno de estos aspectos que se ha dicho que conforman la arquitectura, abre para el arquitecto ricas posibilidades, pero conlleva igualmente, en otro sentido, ciertos límites y peligros. Tanto unas como otros deben ser objeto de conocimiento y reflexión por parte del profesor, de forma personal pero también conjuntamente con los alumnos en el transcurso de su labor docente, en la tarea permanente de repensar su materia.

Así, como artista, el arquitecto es capaz de intuir, alumbrar y hasta de "imprimir" en el espacio -sirviéndose de muy variados elementos materiales y estructuras- dimensiones de la realidad, e incluso del propio ser humano, que pueden permanecer ocultas o inaccesibles a los científicos y a los técnicos.

\begin{abstract}
Como cualquier otra clase de arte, la Arquitectura une lo material con lo espiritual, lo sensible con lo inteligible, lo concreto con lo abstracto. Su "inteligibilidad" consiste en captar tal unión en los nuevos espacios "concretos" [...]. Por tal razón, las nuevas formas espaciales dejan de ser puramente abstractas [como lo son en la geometría]; se imbuyen de sentimientos e ideas humanos [muy probablemente acordes con la preconcepción que del hombre y del mundo tenga el arquitecto] que, por su universalidad, sobrepasan el tiempo y el espacio. (Kupareo, 1969, p. 239) ${ }^{3}$
\end{abstract}

De ese modo, el arquitecto se convierte en "creador" de un orden espacial singular (irreductible al espacio imaginario, al matemático o al físico) y en transfigurador del valor de un lugar.

\begin{abstract}
Es así como una casa, un lugar para ser habitado, puede encarnar ideas de unidad familiar, intimidad, defensa, alegría, comunión con la naturaleza, etc. Una iglesia, lugar de oración, puede sugerir ideas de comunidad de los fieles, presencia de un Ser Absoluto, de auditorio para la Palabra de Dios, meditación íntima, júbilo, austeridad evangélica, etc. Una escuela, lugar de enseñanza, puede sugerir la prolongación del ambiente familiar, convivencia humana, etc. Y así [igualmente] se puede elevar la función de los estadios, de las fábricas, de los hospitales, de las cárceles etc. (Kupareo, 1969, p. 31)
\end{abstract}

Ahora bien, como se ha dicho, el arquitecto debe también ser consciente de que no es solo un artista. Su arte no es, valga la expresión, un "arte puro"; su oficio no es el del arte por el arte, sino que tiene una primera función muy precisa y de carácter eminentemente práctico, utilitario. Su creación no existe solo para ser contemplada sino para ser habitada: y aquí se trata de dar respuesta no únicamente al deseo de belleza que existe en el hombre, sino -en primera instancia- a su necesidad de un abrigo y un refugio seguros, firmes, estables, cómodos; e incluso a lo que el mundo y la sociedad del momento le están reclamando. Pues, en realidad, el arquitecto no crea de la nada, "sus acciones se articulan con el mundo cultural y social que lo rodean, al

3 Dicho sea de paso, esta intuición de que detrás de lo concreto del edificio hay algo más abstracto y, a la vez, más humano, explicaría que incluso las ruinas nos transmitan algo. 
cual pertenece -como conciencia colectiva-, la naturaleza que lo soporta -territorio- y su ubicación dentro del mundo natural donde se localiza -geografía, topografía- y el lugar construido como preexistencia espacial y formal" (Correal Pachón, 2008, pp. 64-65).

Además de su mundo interior, el arquitecto

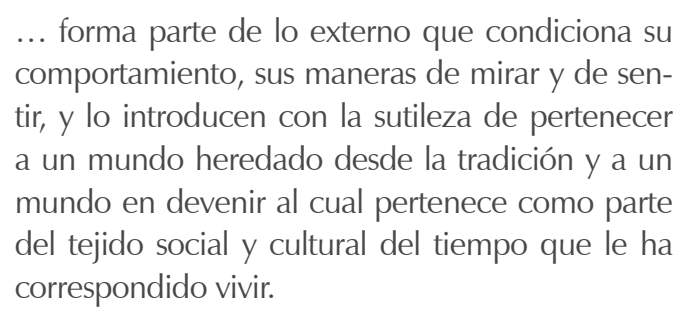

De este modo, el arquitecto teje y entreteje los elementos de conocimiento de la realidad extra-subjetiva con los elementos propios de su creación formal. (Correal, 2008, p. 65)

Dicho de otro modo, el componente artístico de la Arquitectura no debe ser nunca un obstáculo para su correcta, fiable y duradera ejecución física, sino más bien un reto de superación y prolongación de esta.

A estos otros aspectos son a los que se refieren las dimensiones científica y técnica de la Arquitectura. Por una parte, en efecto, en su proceso creador el arquitecto se ve "atado" por procedimientos prestablecidos e incluso por normas tecnológicas de urbanismo y edificación que se hallan recogidas en los distintos sistemas legales elaborados a tal efecto (a menudo, como es sabido, dependen de aspectos tales como la geografía y la geología del lugar, su exposición a movimientos sísmicos, la cultura y el modo de vida propio de la idiosincrasia local, la densidad de población y lo exiguo del espacio urbanizable, etc.). Se trata de armonizar los requerimientos técnicos y las demandas del mercado con las finalidades y necesidades humanas, pero esos requerimientos y demandas existen y actúan como límite a la creatividad del arquitecto, que se ve así desafiado en su dimensión de artista al tiempo que, en otro sentido, busca servirse de los avances científicos para el empleo de nuevos materiales que le abran a nuevas posibilidades técnicas y a nuevas formas estéticas. Se advierte aquí, una vez más, esa especie de dialéctica -limitante pero también fecunda- entre lo material y lo espiritual, entre lo sensible y lo inteligible, que ya antes se pudo percibir.

Por otra, como se acaba de apuntar, se trata de hacer un trabajo profesional que perdure, responda a las exigencias del cliente y garantice la mayor seguridad posible de la obra. Pero, para ello, el arquitecto debe dominar la disciplina arquitectónica, su metodología, y las ciencias que para el desempeño de su oficio le son imprescindibles como apoyo y "alimento" de su quehacer (matemáticas, física, geología, botánica, informática, etc.).
En relación con las tres dimensiones de la arquitectura mencionadas (arte, técnica, ciencia) sería idóneo, parafraseando el lema de un famoso filósofo, "distinguir para unir", pues los peligros de reducir una dimensión a otra, o de priorizar alguna de ellas en perjuicio de las demás siempre están presentes. Es obvio, no obstante, que el menor de los peligros -habida cuenta del carácter utilitario que, salta a la vista, la arquitectura no puede soslayar de ningún modo- es el de priorizar la dimensión artística. Es mucho más factible, en cambio, que el quehacer del arquitecto se oriente hacia posiciones unilaterales científico-técnicas, que beban de una mirada mecanicista e ingenieril sobre la realidad y sobre sí mismo, un enfoque racionalista, pragmático, materialista y, por ende, reduccionista, que lo lleve a contemplar la realidad bajo el prisma exclusivo de su especialidad (Figura 3).

El siguiente punto de este trabajo se dedicará a este riesgo, que no solo afecta seriamente al modo en que uno proyecta, diseña y crea, sino que puede generar en el arquitecto, igualmente, un estilo muy determinado de enseñar e incluso de vivir.

\section{La tentación cientificista y el mito de la inmediatez}

Hablando en términos generales, es evidente que el estudio científico experimental, en su propósito de dar cuenta de la realidad desde una perspectiva y con una metodología muy concretas, lejos de abrirse sin mediación alguna a toda la realidad, prejuzga y orienta los problemas, tanto en su planteamiento como en su resolución, en la línea particular de la causalidad eficiente material ${ }^{4}$. Como ha dicho el físico, filósofo y teólogo Mariano Artigas:

\footnotetext{
El enfoque de las ciencias experimentales supone un punto de vista en el que está predeterminado el tipo de cosas que se pueden decir y, por tanto, el tipo de entidades que se pueden encontrar. Concretamente, la ciencia experimental no se extiende, por principio, a las realidades espirituales; por tanto, negar el espíritu en base a esas ciencias es un cientificismo insostenible. (1984, p. 392)
}

¿Qué quiere decir Artigas con esta observación? Del cientificismo se pueden dar muchas definiciones. Según el filósofo de la ciencia Gerard Radnitzky, "es la creencia dogmática de que el modo de conocer llamado ciencia es el único que merece el título de conocimiento; y su forma vulgar, la creencia de que la ciencia resolverá eventualmente todos nuestros problemas o,

\footnotetext{
4 En este punto, se hablará en paralelo de ciencia y técnica. No son lo mismo e incluso puede cuestionarse que quepa definir sin más la técnica, como frecuentemente se hace, en términos de "ciencia aplicada". Pero, a pesar de ello, no parece que se cometan errores de gravedad si se presume que buena parte de lo que se diga aquí desde el punto de vista epistemológico para la ciencia puede proyectarse también -con matizaciones y cuidado- para la técnica.
} 


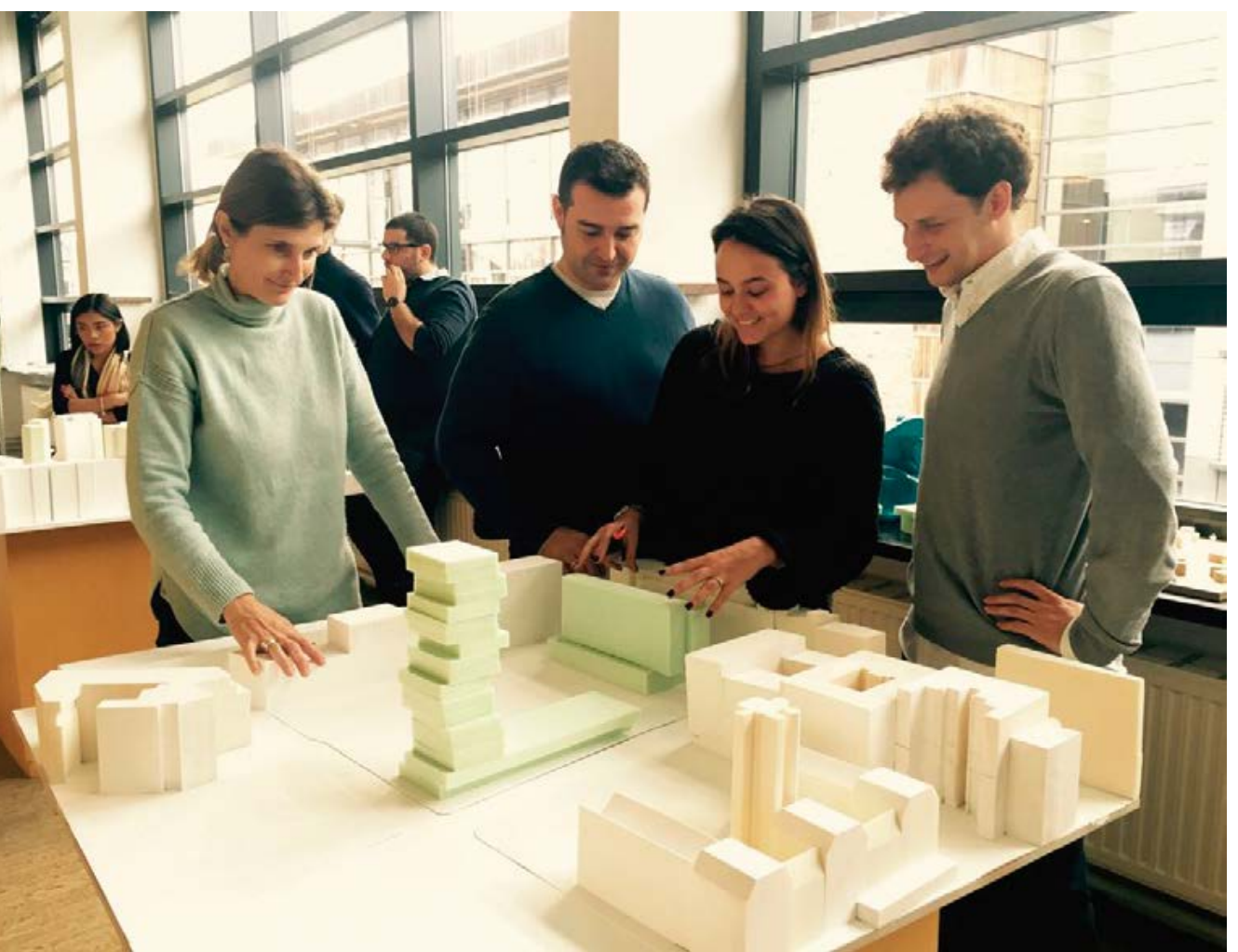

(A) Figura 3. Workshop, Escuela de Arquitectura UFV Fuente: elaboración propia, 2018 cuando menos, todos nuestros problemas significativos" (1973, pp. 254-255).

Paradójicamente, el cientificismo es-en sí mismo- una concepción filosófica, no científica, que rara vez explicita sus presupuestos y aún menos veces aún intenta justificarlos. Sin embargo, puesto que se presenta como una forma de explicación relativamente asequible y capaz de dar razón de todo lo real, no deja de ser una dulce tentación tanto para los científicos de las diversas especialidades como para los técnicos e ingenieros.

No se debe pensar que se trata de cuestiones inocuas y alejadas de la realidad. En primer lugar, como apuntaba Artigas, una concepción exclusivista del saber científico y tecnológico tiene ineludibles consecuencias sobre el modo en que el hombre se interpreta a sí mismo y a los otros.

En segundo lugar, desde esa interpretación, tanto la ciencia como el científico se ven incapacitados para mantener una cierta coherencia interna que evite la división (en sí mismo y respecto de los diferentes niveles que componen su vida), así como para dialogar con disciplinas y personas que empleen una metodología diferente (por ejemplo, la filosofía, el arte o el resto de los estudios humanísticos). Es lógico. De acuerdo con este modo de ver las cosas, solo la ciencia experimental o aplicada podría aún aspirar mínimamente a erigirse en paradigma de fiabilidad, pero sobre todo de utilidad y eficacia; en definitiva, de lo que hoy se entiende por progreso. En cambio, en el resto de los "saberes" anidarían la subjetividad y la duda, la inutilidad y la ineficacia, $y$-en fin- la incapacidad para un progreso real.
Dadas estas condiciones, y a pesar de que la mayoría de los científicos y tecnólogos profesionales ya no suelen incurrir por regla general en este tipo de ilusiones, puede suceder $-y$ de hecho forma parte de la mentalidad más común hoy, contagiada de una vulgarizada cosmovisión tecno-científica que tiene muchos rasgos propios de una verdadera ideología- que se proceda a una inaceptable "mecanización de nuestra visión del mundo" y del propio ser humano, debida a la falsa creencia de que las ciencias son absolutamente objetivas, y a la "errónea suposición epistemológica de que el uso de un esquema interpretativo para extender nuestra comprensión excluye el uso de los demás" (Boden, 1984, p. 610). Que la ciencia o la tecnología sean, obviamente, diferentes de la poesía o del mito, no significa que los pueda excluir. $\mathrm{O}$, en el caso que aquí se trata, que la arquitectura requiera de técnicas precisas, del conocimiento de las propiedades de los materiales que emplea y de las leyes científicas que rigen su dinamismo; en definitiva, de todo aquello de lo que depende su ejercicio, no significa que se pueda obviar su dimensión artística.

Por último, cuando un esquema como el descrito se impone, no resulta difícil perder de vista también toda perspectiva: hasta los criterios morales más simples se pueden postergar en beneficio del rendimiento, la economía, la utilidad o un desarrollo dudosamente al servicio del hombre y -con frecuencia- en perjuicio de la naturaleza. No se trata solo de que, como afirmó el famoso filósofo de la tecnología Carl Mitcham, tal vez no sea un accidente "que una sociedad dominada por la racionalidad técnica e instrumental deba ser marcadamente utilitaria en sus preceptos culturales" (1989, p. 119); lo peor es que, cuando en lugar de contemplar que el primer y más importante valor de los actos humanos (no el único) reside en ellos mismos y que, precisamente por ello, las acciones que el ser humano realiza pueden ya dignificarlo o corromperlo, se atiende única y exclusivamente a sus consecuencias prácticas (en términos mecanicistas y pragmáticos que reducen las operaciones que se llevan a cabo al hecho de que "funcionen" o de que proporcionen el máximo beneficio material posible), es señal de que se ha empezado a perder el rumbo y se avanza hacia un desarrollo no sostenible y desnortado, que difícilmente puede ser considerado, por tanto, como un verdadero progreso.

De lo dicho se deduce que, cuando en su práctica y en sus reflexiones, algunos científicos o tecnólogos no respetan las limitaciones inherentes a sus propias disciplinas, incurren en un inaceptable reduccionismo que acarrea implicaciones muy negativas. A este respecto, las observaciones del filósofo de la ciencia Karl Popper (1996) resultan son muy oportunas. 
Distingue este autor entre un reduccionismo metodológico y un reduccionismo filosófico. El primero no es otra cosa que el tipo de aproximación analítica propia del método científico, indispensable en el ejercicio de la ciencia. El segundo, en cambio, completamente rechazable al parecer del que esto escribe, consiste en usar como guía, luz y clave interpretativa de la praxis experimental una determinada posición ideológica, en este caso una creencia cientificista y, por ende, materialista.

Popper lo ha explicado de este modo:

En el transcurso de esta exposición defenderé tres tesis. En primer lugar, sugeriré que los científicos tienen que ser reduccionistas en el sentido concreto de que no existe mayor éxito en la explicación científica que el de una reducción lograda [...]. En segundo lugar, sugeriré que los científicos tienen que aceptar de buen grado el reduccionismo como método, ya sea de forma ingenua o más o menos crítica; en realidad, en mi opinión, serían unos reduccionistas críticos un poco a la desesperada, porque apenas ha habido alguna reducción científica de importancia que haya tenido un éxito total [...]. En tercer lugar, sostendré que no parece haber ningún buen argumento a favor del reduccionismo filosófico, mientras que -al contrario- hay muy buenos argumentos contra el esencialismo con el que el reduccionismo filosófico parece estar estrechamente ligado. (1996, p. 154)

Así pues, el reduccionismo metodológico es razonable e incluso exigible pues la ciencia $-y$ lo mismo se puede decir aquí de la tecnología-, para proceder de acuerdo con su naturaleza y objetivos se ve obligada a simplificar de algún modo la realidad para que pueda entrar en sus cánones analíticos o procedimentales. Incluso es posible que en ciertas ocasiones tenga que prescindir consciente y circunstancialmente $(\mathrm{o}, \mathrm{al}$ menos, no darles toda la fuerza y la consideración quizás deseables) de aquellos datos ofrecidos por la experiencia que no se dejen canalizar por las vías que les son propias (piénsese, por ejemplo, en la experiencia personal o en la experiencia común). Pero precisamente porque se trata de una reducción -que, idealmente, se lleva a cabo con matices y plena conciencia- los científicos y tecnólogos tienen que extremar el cuidado en no extralimitarse en sus interpretaciones desembocando en lo que algunos han Ilamado muy apropiadamente el "nadamasqueísmo".

Como Aldous Huxley dijo hace ya bastante tiempo, pragmáticamente los científicos (mutatis mutandis se podría decir algo paralelo en el caso de los técnicos) están justificados al actuar de:

... forma tan caprichosa y arbitraria; porque al concentrarse [...] en los aspectos mensurables [o manejables] de los elementos de la experiencia que pueden ser explicados en términos de un sistema causal [eficiente y de orden material], han podido conquistar un dominio creciente sobre las energías de la naturaleza. Pero poder no es lo mismo que intuición y, como representación de la realidad, la descripción científica del mundo es inadecuada, por la simple razón de que la ciencia ni siquiera pretende tratar el total de la experiencia, sino solo algunos de sus aspectos y en determinadas relaciones. Todo esto lo entienden claramente los hombres de ciencia dotados de espíritu más filosófico.

Pero infortunadamente algunos científicos, muchos técnicos y la mayoría de los consumidores de sus invenciones carecen del tiempo y de la inclinación para examinar las bases filosóficas de las ciencias. En consecuencia, tienden a aceptar la descripción del mundo implícita en las teorías científicas como una explicación completa y exhaustiva de la realidad, tienden a considerar aquellos aspectos de la experiencia que los científicos, no sintiéndose competentes para ello, no toman en consideración, como algo menos real que los otros aspectos que la ciencia arbitrariamente decide abstraer de entre la infinitamente rica variedad de hechos dados. (1963, pp. 45-46) ${ }^{5}$

Sin embargo, en realidad, ni una confirmación experimental viene a ser por sí sola una verdadera prueba de una tesis metafísica, a no ser que se prejuzgue que el campo de lo físico agota todo lo real; ni ser capaz de fabricar o construir algo tiene por qué significar que se tiene verdadera comprensión de su naturaleza, salvo si uno adopta -por opción o prejuicio- una perspectiva epistemológica radicalmente pragmática, que reduzca la verdad al éxito en las aplicaciones de una hipótesis.

Como ha hecho notar el físico Marco Bersanelli:

En las ciencias experimentales la certeza, cuando sucede, normalmente es el fruto de muchos resultados convergentes; nace de la acumulación de numerosas e independientes observaciones a lo largo del tiempo; es el resultado de indicios diversos, ninguno de los cuales es definitivo por sí solo, sino que la conjunción de todos ellos pone a la razón delante del nacimiento de una propiedad específica de lo real. En este sentido, para un investigador cada experimento es la búsqueda de un signo. Existe un punto en el cual la razón, frente a la complejidad de los "signos" acumulados en relación a un cierto fenómeno, puede reconocer positivamente "cómo son las cosas" o más exactamente "cómo han sido hechas": desde luego no con la absurda pretensión de definir exhaustivamente el objeto, sino con el estupor agradecido por la toma de conciencia de un fragmento de la realidad creada.

Pero, incluso frente a una multitud de signos, el brote de la certeza no está fijado mecánicamente y no está escrito en una fórmula o algoritmo. La capacidad de reconocer como único sentido razonable aquel punto sobre el cual converge el conjunto de todos los indicios, es propia solo del yo humano. El conocimiento científico, como todo conocimiento, consiste en un encuentro entre el nivel supremo de lo real, el sujeto humano autoconsciente, con otro aspecto de la realidad [o de

5 A este respecto, algún autor ha advertido, muy agudamente, que reducir suele ser un procedimiento muy socorrido para suprimir misterios. (Chalmers, 1999) 
campo de lo posible] (una piedra, un abedul, la tierra, otro ser humano [o, añadimos, un proyecto arquitectónico]). Sin el sujeto de conocimiento no existe conocimiento: es verdaderamente vertiginoso el papel que le ha sido confiado al ser humano dentro del gran escenario de la creación. Por ello, toda concepción que reduzca el campo de la razón y debilite u olvide el yo humano, está condenada al final a la duda sistemática y al rechazo de toda forma de certeza y por lo tanto de toda conquista real (1999, párrs. 9 y 10)

Recuperar, entonces, la dimensión personal del conocimiento no riñe con admitir su falibilidad y, al mismo tiempo, su fiabilidad a la luz de la acumulación de indicios. Lo que sí se opone a esta dimensión personal del conocer es la utopía cientificista que, partiendo de una reducción de lo real metodológicamente necesaria, pretende borrar el hecho de que la interpretación científica de la realidad es una forma de mediación, y sujeta, entonces, a críticas parecidas a las que se suelen dirigir a otras formas más comunes e inespecializadas de interpretar la realidad.

\section{Discusión}

\section{La arquitectura desde la razón ampliada}

En consecuencia, si se quiere encontrar una concepción capaz de hacer justicia tanto a la realidad en su globalidad como a la triple dimensión (artística, científica, técnica) de la arquitectura, se debe poner en práctica lo que -siguiendo al teólogo Josef Ratzinger- puede llamarse una "razón ampliada"; perspectiva que, a su vez, implica adoptar una posición realista en el orden epistemológico.

Este realismo, al que este trabajo se va a referir ahora brevemente ${ }^{6}$, no merece adhesión por una cuestión de oportunidad, conveniencia o simpatía personales, sino porque -en cuanto que perspectiva epistemológica- parece la posición mejor fundada a la luz del análisis filosófico; la que da razón de un modo más sólido y completo del conocer humano en todas sus dimensiones y canales, salvaguardando la consistencia de lo real que la explicación científica exige y sobre la que toda tecnología y todo arte se apoyan para dar lugar a nuevas creaciones, a partir de la transformación de materiales previos, y en concordancia con una idea o diseño previamente pensados y proyectados; la que ofrece un punto de apoyo más coherente y rico para la vida; la más acorde con la experiencia común y, en definitiva, la que se podría considerar como posición "natural" de la inteligencia humana.

El "realismo crítico" (así puede ser denominado siguiendo a algunos eminentes pensadores

6 Lo que aquí sigue en relación con los modos plurales y diversos del conocer humano está tomado de Álvarez-Álvarez (2011). contemporáneos) reconoce la pluralidad y heterogeneidad de lo real al mismo tiempo que busca profundizar todo lo que sea posible en su conocimiento. Acepta las limitaciones de las capacidades humanas respecto de un conocer que siempre será limitado en extensión y parcial en su contenido. Pero también, la posibilidad de que ese conocimiento sea verdadero y progresivo. Para ello, tendrá en cuenta los diferentes planos que conforman la realidad, los distintos rasgos y grados de dinamismo y estructuración que conforman -ya en primera instancia- a los seres que se encuentran al alcance de la experiencia humana (articulados en "sistemas" gradualmente más extensos y complejos), y el singular dinamismo y la peculiar estructuración de la condición que sitúa al ser humano en el punto de partida de lo que puede llegar a ser y conocer.

Todo ello, desde el punto de vista epistemológico, implica -por una parte- el sentido profundamente relacional (no relativista) de cualquier realidad y de cualquier forma de conocimiento humano, incluso su dimensión contextual y parcial, pero también la consistencia propia y la inteligibilidad de las realidades que queremos conocer; y, por otra, la necesidad de orientar nuestra reflexión hacia fines, y el uso de medios de investigación, muy diferentes y más flexibles que los planteados por un concepto reduccionista de la ciencia y de la razón.

En definitiva, a la pregunta de cómo puede el hombre conocer la verdad de las cosas y de sí mismo habría que responder: sabiendo descifrar el mapa de la realidad, es decir, utilizando en cada caso un método adecuado al objeto que se quiera investigar y a través de los diversos canales o tipos de conocimiento con los que cuenta. El conocimiento, en efecto, no es una realidad unívoca (no se aplica el término "conocimiento" a las distintas formas de conocer según un significado idéntico en todos los casos) sino análoga (se aplica a los diversos modos de conocer: ver, tocar, entender etc., de acuerdo con un significado que es en parte semejante pero también en parte diferente). Por eso, si se quiere ser justo y riguroso se deben distinguir y valorar en su justa medida esos diversos modos del conocer humano.

Sin ánimo de ser exhaustivos sobre esta cuestión, en principio se podría hablar de dos formas de conocimiento humano: la razón y la fe. Efectivamente, las certezas y convicciones humanas no siempre se apoyan sobre un conocimiento objetivo (un conocimiento que hace patente, de algún modo, lo que se conoce o su verdad; lo que habitualmente se llama conocimiento racional), sino que, en múltiples ocasiones (y el ámbito científico-tecnológico no es una excepción), se sostienen sobre la veracidad y credibilidad de un testigo (de alguien que sí ha conocido por vía 
de saber), y que transmite una noticia a la que el sujeto -por el motivo que sea- no tiene acceso, y a quien este se adhiere por ser aquel quien es.

Además, cabe distinguir -en un segundo momento- entre conocimiento objetivo o conceptual y conocimiento por connaturalidad e inclinación. Este último es un conocimiento que tiene lugar en el intelecto, pero no en virtud de relaciones puramente conceptuales y por vía de estricta demostración. En él, la inteligencia obra conjuntamente con tendencias afectivas y disposiciones de la voluntad que la guían y dirigen. Sus funciones más propias son, ciertamente, las del dominio del obrar, de la prudencia y de la poesía, pero también todo el inmenso campo de certezas que, si para muchos no llegan a merecer el calificativo de "científicas", enriquecen no obstante la vida humana; más aún, sin las que el hombre no puede realmente vivir.

Se dirá que este tipo de conocimiento -individual y difícilmente comunicable- no es requerido de forma intrínseca para la actividad natural de la inteligencia que es el progreso de la ciencia, pero -incluso dando por supuesto que así fuera- eso no significa que no ocupe un lugar muy importante en el conocer y en la vida humana (particularmente en el campo del conocimiento artístico y la experiencia estética, del conocimiento moral o de la experiencia religiosa), que no pueda proporcionar sus propios elementos de juicio en el plano de la razonabilidad o que no sea capaz de dar lugar a una cierta intersubjetividad. Además, es esencial a la hora de dar razón de la actividad creativa del espíritu humano.

En cuanto al "conocimiento por modo de conocimiento", conceptual y demostrativo, el especulativo, o simplemente el conocimiento en el sentido más habitual del término, en el orden estrictamente científico es el que tiene el papel protagonista. Pero aquí caben también algunas otras distinciones.

Por una parte, cabe diferenciar entre conocimiento intuitivo y conocimiento discursivo ${ }^{7}$. El primero se define por la presencia (intencional) de un objeto a una facultad (ya sea desde un punto de vista sensible o intelectual, pero en cualquier caso con un carácter de inmediatez y evidencia objetiva). El segundo consiste en un movimiento del espíritu que pasa de un conocimiento a otro, obtenido por medio de aquel, y se refiere -de forma fundamental, aunque no única- al encadenamiento de proposiciones que constituyen los razonamientos.

7 Se emplea aquí el término "intuición" en un sentido distinto al usado hasta el momento. Al tratar de la arquitectura, fue enmarcado en el orden del "conocimiento por connaturalidad"; ahora, pertenecería al conocimiento especulativo. Mientras que, en este, la intuición presenta el objeto con evidencia inmediata, y da así lugar a una certeza absoluta e incondicionada, en aquel genera otra forma de certeza, hipotética y condicionada pero no por ello menos relevante para el ser humano.
Por último, en otro sentido más común, se suele distinguir entre experiencia y razón. Se dice, desde esta perspectiva, que el conocimiento humano tiene dos dimensiones que están íntimamente relacionadas: la sensible y la intelectual, y ambas se hallan igualmente presentes en todo saber y en el modo de conocer humano, a veces -por así decir-predominantemente sensible; en otras ocasiones, predominantemente intelectual.

Como se ve, el conocimiento humano resulta ser una estructura dinámica y procesual, a la vez unitaria y compleja. En realidad, no hay un único modo de conocer, pues quien conoce es la persona entera, y todos esos canales de conocimiento contribuyen a perfeccionarla y la ayudan en su búsqueda de la verdad acerca de una realidad que es igualmente plural y compleja. No sirve, por tanto, para hacer justicia a la riqueza y pluralidad de lo real, lo mismo que a la riqueza de nuestro propio ser, un concepto estrecho y reductivo de razón como el que se ha considerado al principio de este trabajo. Se precisa de un concepto de razón "ampliada", correspondiente con la integridad del ser humano y con las múltiples dimensiones que parecen estructurar la realidad.

\section{Conclusiones y sugerencias finales}

Los resultados de la reflexión llevada a cabo en este trabajo parecen claros. Desde una razón ampliada, la arquitectura no puede comparecer exclusivamente en su dimensión técnico-científica. Y tampoco su enseñanza, entonces, puede pretender seguir ese estrecho camino. Si, además de ciencia y técnica, se está ante una de las bellas artes, será pues responsabilidad de los profesores el ser capaces de integrar las dimensiones de la arquitectura con su propia experiencia, para así poder enseñarla de tal manera que se produzca el milagro de la transmisión educativa, que se da cuando dicha comunicación queda impregnada de la experiencia pasional, de ese pathos que arrastra a todo aquel que se enamora de su saber y también a quienes lo escuchan.

No parece oportuno terminar estos apuntes sin un breve añadido final, alimentado y acompañado de algunas citas, que incluye algunas sencillas explicaciones sobre lo que constituye el objeto y fin de una disciplina, y la formulación de tres preguntas que puedan servir como pistas para el repensamiento de la arquitectura en lo que tiene que ver con la cuestión epistemológica.

Primero, sobre el objetivo último de este repensamiento, cabe en primer lugar recordar la advertencia mencionada al comienzo de este artículo. El fruto buscado no es, desde luego, la conformación de un plan de estudios híbrido consecuencia de la yuxtaposición de materias propias de los estudios en Arquitectura, con otras asignaturas de carácter humanístico; tampoco, la mezcolanza, en el seno de cada disciplina, de 
conceptos filosóficos o teológicos junto con los científico-técnicos. No se trata, como advierte María Lacalle Noriega,

\begin{abstract}
... de que el profesor de Matemáticas o el de Estructura de materiales expliquen Metafísica o Teología en sus clases, sino de que, en sus explicaciones, en su trato con los alumnos, en su comunicación con ellos y a partir de los contenidos de la materia [o incluso de lo que la propia experiencia universitaria del alumno y el profesor, en su relación mutua, pueda suscitar], les remitan a las preguntas fundamentales, a la verdad, al bien, al porqué de las cosas. Se trata de replantear cada una de las asignaturas superando los límites de cada área de conocimiento, ampliando horizontes y buscando en nuestra enseñanza una dimensión mucho más profunda. (2014, p. 32)
\end{abstract}

Lo que se ha de interpretar en este texto es que, aunque no todas las asignaturas permiten igual profundidad en el repensamiento, siempre es posible reconocer tanto las posibilidades que las distintas disciplinas ofrecen como los límites -e incluso los peligros latentes- que les impiden contribuir a que el ser humano avance en la búsqueda de verdades cada vez más profundas y trascendentes, verdades referidas al porqué de las cosas, al buen obrar y al sentido de las acciones, a una vida lograda, etc. Obviamente, eso no significa que el arquitecto deba ser al mismo tiempo sociólogo, filósofo, politólogo, economista o hasta teólogo, pero sí que comprenda qué hay más allá de su ciencia, pero en conexión con ella, y que -como persona y como profesor- tenga la inquietud por formarse al respecto. De esta manera, podrá satisfacer auténtica y plenamente las necesidades de aquellos a los que atiende como arquitecto, también él, buscador infatigable pues tiene la pretensión de descubrir todo aquello que permita cumplir su misión completamente, y servidor de los otros por su empeño en colaborar con el bien de las personas y las sociedades.

Segundo, para que tenga el debido realismo y pueda ser realmente eficaz, el proceso de repensamiento de cada asignatura no puede detenerse en esta tarea meramente reflexiva actitudinal. Debería culminar en la elaboración de un syllabus detallado que incluya el objeto y el fin de la asignatura, las competencias que se pretenden desarrollar, los contenidos, la metodología, los materiales y recursos que se considera emplear, y hasta el sistema de evaluación.

Desde la perspectiva epistemológica objeto de consideración en este artículo, ża qué se refiere este párrafo cuando habla del objeto, método y fin de la asignatura? A toda ciencia, particularmente en los momentos de su fundación y precisamente para dotarla de una constitución adecuada, se le plantea la necesidad de establecer un objeto de estudio acotando una parcela de la realidad que hace suya: es lo que, clásicamente, se dio en llamar "objeto material" de un saber. Pero también, paulatinamente y a medida que ese saber progresaba, de forma casi ineludible experimentaba la necesidad de considerar de un modo más preciso y riguroso la determinación y especificación de su objeto, para distinguirse de otros saberes que podían surgir en el interior de la propia disciplina o en zonas fronterizas a ella: es lo que se llamó "objeto formal".

Así pues, aunque puede haber ciencias distintas que tengan el mismo objeto material, por este se entiende el conjunto de cuestiones relativas a una determinada parcela o nivel de lo real que, por así decir, son "materia" de una ciencia. Por su parte, el objeto formal de un saber es aquel que caracteriza propiamente a cada ciencia dotándola de una cierta autonomía respecto de aquellas otras que les son más o menos anejas, lo que le da su último toque (en su enfoque específico y en la metodología acorde con él). Dicho de otro modo, es la perspectiva o punto de vista desde la que se estudia el objeto material, y determina en un primer sentido el modo de aproximación a través del cual se ha de afrontar ese objeto de estudio.

Un ejemplo ayudará a entender estas distinciones. Las Ilamadas "ciencias humanas" tienen como objeto material de estudio al hombre. Pero, obviamente, es muy diferente la historia, de la psicología, de la pedagogía o de la biología humana. Estos saberes se distinguen por su objeto formal: la historia analiza al ser humano en cuanto que ser histórico, que vive y actúa en un tiempo; la psicología lo analiza desde la perspectiva de su psique o mente (y de los actos "psíquicos"); la pedagogía, en cuanto sujeto de aprendizaje; la biología lo analiza en cuanto que ser vivo. Cada uno de esos enfoques determinará la metodología que habrá que emplear, el grado de abstracción en que los conceptos propios de ese saber se mueven, la forma en que se verificarán sus conclusiones, etc., Pero icuidado!, ninguno de ellos por separado podrá pretender dar razón completa del ser humano. Si lo quisiera hacer así, incurriría en lo que hemos Ilamado "reduccionismo". Y si, dado el prestigio que hoy tiene la ciencia, fuera la biología humana la que quisiera ejercer ese reduccionismo pretendiendo explicar por completo todo lo que el ser humano es, e incluso imponiendo su método a los otros saberes mencionados de manera que si no se adaptaran a esa metodología científica se entendería que carecen de valor, estaríamos en lo que se denomina "cientificismo".

Por último, el fin no es más que lo que se quiere conseguir con el tratamiento científico del objeto material, aplicando los métodos propios de la ciencia en cuestión y teniendo en cuenta 
el enfoque desde el cual se estudia dicho objeto material (objeto formal).

En el caso de la arquitectura, se podría decir -recapitulando cosas ya mencionadas- que su objeto material es el espacio. En ello es coincidente con otras disciplinas como la geometría (y por eso precisamente hay una cierta relación entre ellas). El objeto formal, es decir, el punto de vista desde el cual se afronta ese objeto material, sería el espacio considerado -sobre el terreno- como dimensión de lo real. También aquí hay coincidencias con otras disciplinas como, por ejemplo, la geotecnia (y por eso conviene estudiar los fundamentos básicos de esta ciencia); pero en el caso que nos ocupa, de lo que se trata es de -mediante un proyecto elaborado al efecto- transformar ese espacio, reordenarlo, urbanizarlo, rehabilitarlo, etc. Esta especificación determina que la metodología del arquitecto sea distinta de la del geotécnico. Por último, el fin de la arquitectura es el de hacer de esos espacios lugares habitables para el ser humano, con todo lo que ya sabemos que ello implica. Y también este fin ayuda a perfilar definitivamente el método -o medios- que se ha de seguir para alcanzar aquel.

Tercero, cómo llevar el repensamiento a su último escalón, el del temario o guía docente y el día a día de la clase en cada una de las asignaturas es algo que no puede ser tratado de forma general y en un artículo de esta índole. Solo cabe aquí proponer algunas sencillas preguntas dirigidas a los distintos profesores que imparten las diversas materias, que quizás podrían servirles de pista en el caso de la cuestión epistemológica que ha sido objeto de este estudio, y con las que este termina.

En relación con la asignatura que imparte: ¿cuáles cree que son sus límites a la hora de confrontar sus descubrimientos o aplicaciones con la amplitud de lo que el hombre, en su búsqueda de la verdad, quiere alcanzar? ¿Reconoce esos límites en la impartición de su docencia ante los alumnos, de manera que ellos tengan claro lo que pueden y no pueden esperar de dicha asignatura?

En relación con los alumnos: ipropone a sus alumnos que, en su búsqueda de la verdad, no se conformen con el limitado campo de verdades que su asignatura puede proporcionarles? ¿Les abre a otras dimensiones propias de su condición universitaria y humana? ¿Les muestra la necesidad de que, para ello, reconozcan la diversidad de lo real y de los distintos canales de conocimiento con los que el hombre cuenta para conocer esa multiforme realidad?

En relación con la ciencia que sustenta la asignatura impartida: ¿cuál es el objeto (material y formal) de su disciplina concreta? ¿Cuál su metodología? ¿Y su finalidad?

En relación con el repensamiento más general de la asignatura: en una perspectiva ya más amplia, ¿qué relación puede haber entre estos puntos y las cuestiones que, como se señaló al principio, habrían de articular el repensamiento en general: no solo la cuestión epistemológica, también las cuestiones antropológicas, éticas y de sentido?

\section{Referencias}

Álvarez-Álvarez, J. J. (2011). La investigación humanística en una universidad católica, Comunicación y hombre. Revista interdisciplinar de Ciencias de la comunicación y Humanidades (UFV), (7), 223-235. Recuperado de https://comunicacionyhombre. com/article/la-investigacion-humanistica-una-universidad-catolica/

Álvarez-Álvarez, J. J. (2018). Apuntes para el repensamiento de la enseñanza de la Ingeniería Informática. La cuestión antropológica y la necesidad de una Antropología integral. Revista Educación en Ingeniería, 13(26), 101-107. https://doi.org/10.26507/ rei.v13n26.901

Artigas, M. (1984). Máquinas pensantes y conocimiento humano. En Actas del III Simposio de Teología Histórica (7-9 mayo 1984). Confrontación de la teología y la cultura. Valencia: Facultad de Teología San Vicente Ferrer.

Bersanelli, M. (1999). La tierra es redonda, Huellas, 4. Recuperado de http://www.hueIlas-cl.com/arch/april99/ciencia.htm
Boden, M. A. (1984). Inteligencia artificial y hombre natural. Madrid: Tecnos.

Chalmers, D. (1999). La mente consciente: en busca de una teoría fundamental. Barcelona: Gedisa.

Correal Pachón, G. (2008). El proyecto arquitectónico: algunas consideraciones epistemológicas sobre el conocimiento proyectual. Revista de Arquitectura (Bogotá), 10(1), 63-68. Recuperado de https://editorial.ucatolica.edu.co/ojsucatolica/revistas ucatolica/index.php/RevArq/article/view//784

Huxley, A. (1963). Ciencia, libertad y paz, y la doble crisis. Buenos Aires: Editorial Sudamericana.

Kupareo, R., O. P. (1969). Filosofía de la Arquitectura. Aisthesis, (4), 21-32. Recuperado de http://revistaaisthesis.uc.cl/index.php/ rait/article/view/598/553

Lacalle Noriega, M. (2014). En busca de la unidad del saber. Una propuesta para renovar las disciplinas universitarias. Madrid: Universidad Francisco de Vitoria. Recuperado de http:// wordpressawards.camartis.org/test/wp-con-
tent/uploads/2018/05/PDF INTERACTIVO_En_busca_de_la_unidad_del_saber.pdf Mitcham, C., (1989). Qué es la filosofía de la tecnología. Barcelona: Anthropos.

Popper, K. R. (1996). Post scriptum a La lógica de la investigación científica. Vol. II: El universo abierto: un argumento a favor del indeterminismo. Madrid: Tecnos.

Radnitzky, G. (1973). Hacia una teoría de la investigación que no es ni reconstrucción lógica ni psicología o sociología de la ciencia. Teorema: revista internacional de filosofía, 3(2/3), 197-264. Recuperado de http://www.jstor.org/stable/43047671

Samarán, F. y Parada, J. L. (coords.) (2013). Misión. Grado en Arquitectura. Pozuelo de Alarcón: Universidad Francisco de Vitoria.

Universidad Francisco de Vitoria (s. f.). Nuestra misión hoy. Pozuelo de Alarcón: Universidad Francisco de Vitoria. Recuperado de https://institutojohnhenrynewmanufv. com/wp-content/uploads/2018/06/Mision UFV ES.pdf 



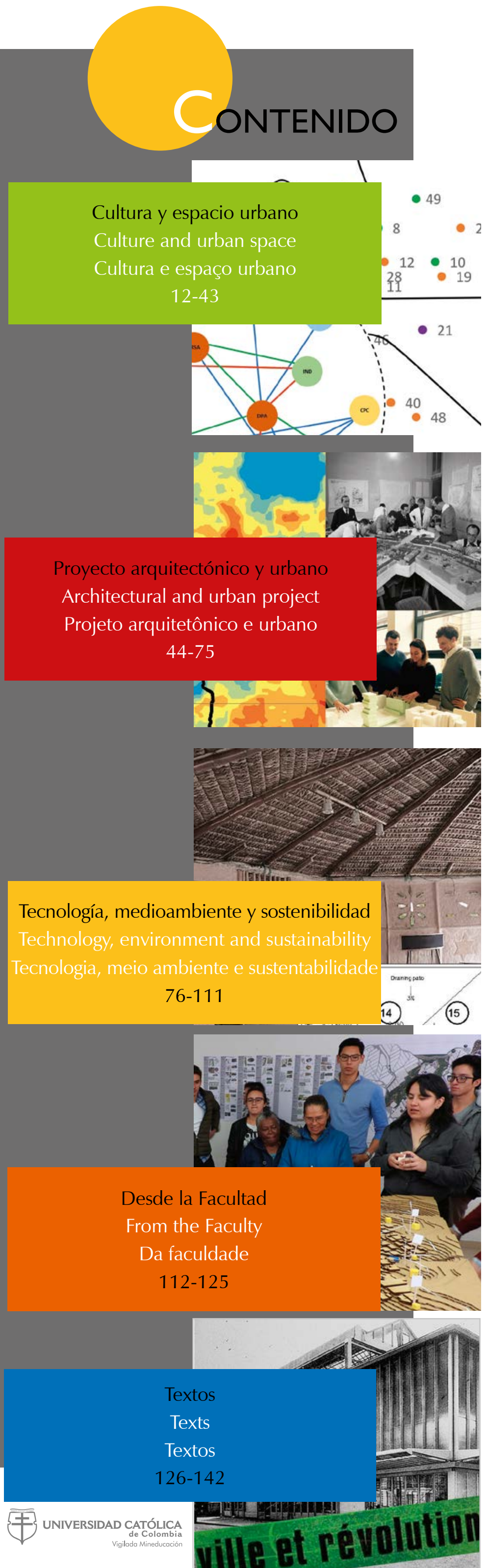

Arquitecturas colectivas y participación como estrategias para la construcción de la ciudad latinoamericana

Myriam Stella Díaz-Osorio

Pág. 3

ES

La caminabilidad en Bogotá: propósitos y condiciones socioespaciales que facilitan y limitan esta experiencia Pablo Páramo

Andrea Burbano

Pág. 12

ES EN

Planificación comunitaria en barrios socialmente

vulnerables. Identificación de los actores sociales en una comunidad

Rafael Alejandro Tavares-Martínez
Jesús Manuel Fitch-Osuna

ES

Pág. 22

Desvanecimiento de la frontera como límite. Imaginario del borde como espacio público físico y virtual

Gabriela Eloísa Muñoz-Torres

Susana Gutiérrez-Luna

Pág. 33

Estudiantes latinoamericanos en el Institut

d'Urbanisme de l'Université de Paris (1923-1941)

$$
\begin{aligned}
& \text { Andrés Ávila-Gómez } \quad \text { Pág. } 44 \\
& \text { ES }
\end{aligned}
$$

Apuntes para el repensamiento de la enseñanza de la Arquitectura. La cuestión epistemológica y la necesidad de una razón ampliada
Juan J. Álvarez-Álvarez
Pág. 57

ES

Equipamientos colectivos: "lugares" de producción de capital social
José Mario Mayorga-Henao
Pág. 68

\section{ES}

Bucle multidisciplinar para la sustentabilidad urbana

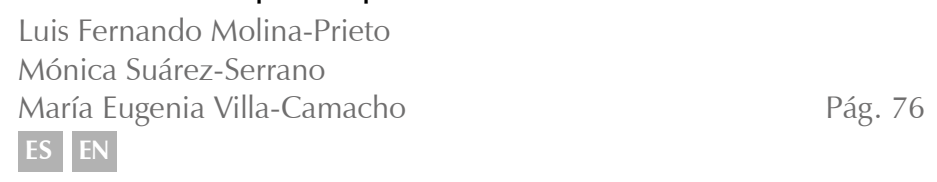

Durabilidad de los materiales naturales de construcción: percepciones de proyectistas, constructores y usuarios en Florianópolis, Brasil

Andrea Salomé Jaramillo-Benavides
Zuleica Maria Patricio-Karnopp
Lisiane Ilha-Librelotto

Pág. 89

\section{ES}

Thermal comfort in buildings for wet processing of coffee

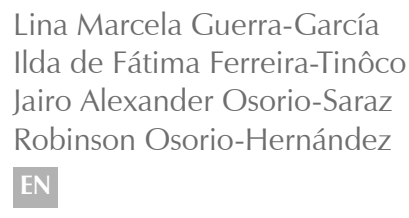

La arquitectura en los barrios: puntos de encuentro entre la academia y el saber popular

Hernando Carvajalino-Bayona

Pág. 112 ES

Arquitectura, modernidad, modernización

$$
\begin{aligned}
& \text { Jean-Louis Cohen } \\
& \text { Traductores } \\
& \text { Andrés Avila-Gómez. } 126 \\
& \text { Diana Carolina Ruiz } \\
& \text { ES }
\end{aligned}
$$


Arquitecturas colectivas y participación como estrategias para la

Arquiteturas coletivas e participação como estratégias para construir a cidad latino-americana

\section{Stella Díaz-Osorio}

La caminabilidad en Bogotá: propósitos y condiciones socioespaciales que facilitan y limitan esta experiencia

The Walkability of Bogotá: purposes and socio-spatial conditions that facilitate and limit this experience

A caminhabilidade em Bogotá: propósitos e condições socioespaciais que facilitam e limitam essa experiência

\section{Andrea Burbano}

Planificación comunitaria en barrios socialmente vulnerables.

Identificación de los actores sociales en una comunidad

Community planning in socially vulnerable neighborhoods.

Identification of social actors in a community

Planejamento comunitário em bairros socialmente vulneráveis.

Identificação dos atores sociais em uma comunidade

Rafael Alejandro Tavares-Martínez

esús Manuel Fitch-Osuna

Desvanecimiento de la frontera como límite. Imaginario del borde como espacio público físico y virtual

$m$

迹

Durabilidad de los materiales naturales de construcción: percepciones de proyectistas, constructores y usuarios en Florianópolis, Brasil

Durability of natural building materials: Perceptions of designers, builders, and users in Florianópolis, Brazil

Durabilidade dos materiais naturais de construção: percepções de projetistas, construtores e usuários em Florianópolis, Brasil

\section{Zuleica Maria Patricio-Karnopp}

Lisiane Ilha-Librelotto

Thermal comfort in buildings for wet processing of coffee

\section{Confort térmico en edificaciones para procesamiento húmedo de café}

Conforto térmico em instalaçōes para processamento úmido de café

\section{Ida de Fátima Ferve-Tartióco}

airo Alexander Osorio-Saraz

Robinson Osorio-Hernandez

La arquitectura en los barrios: puntos de encuentro entre la academia y el saber popular

Architecture in neighborhoods: meeting points between academia and popular knowledge

A arquitetura nos bairros: pontos de encontro entre a academia e o saber popular Hernando Carvajalino-Bayona

\section{Arquitectura, modernidad, modernización}

Architecture, modernity, modernization

Arquitetura, modernidade, modernização
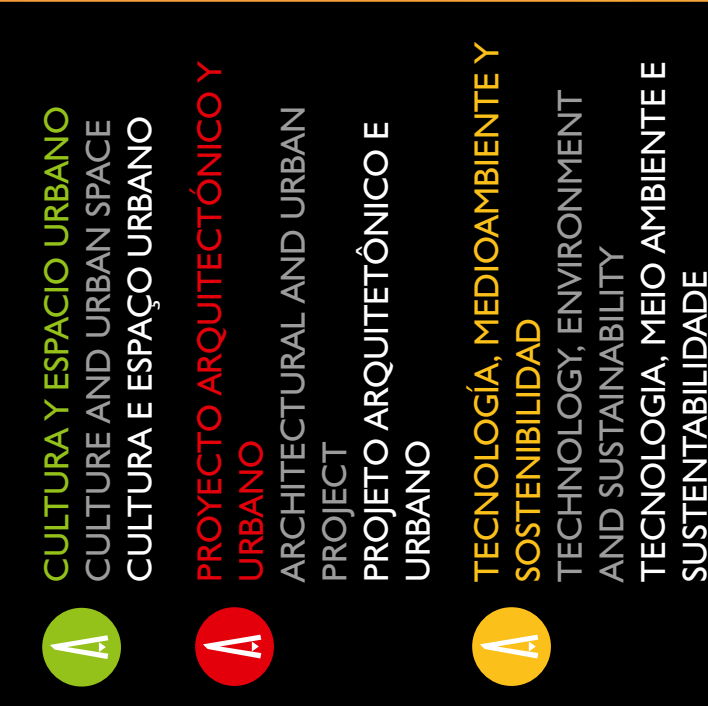

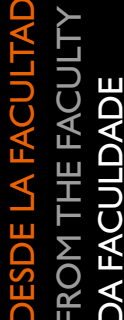

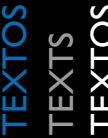
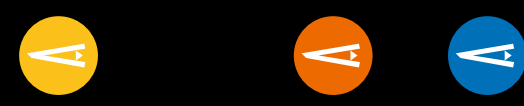

1) Clarivate

publind

Clarivate
Analytics

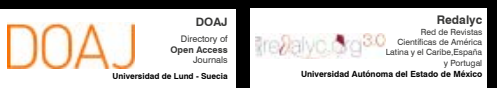

n.

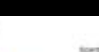

REDIB

EBSCO

cis

latindex unmoxom

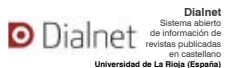

LatinREV

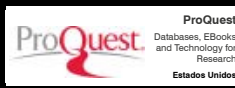

MIคR

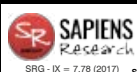

Goosin

Google

ARLA

hi. https://www.mendeley.com/profles/revista-de-arquitectura-bogot/

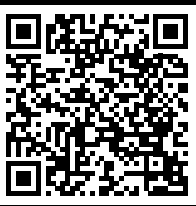

ISSN: 1657-0308 